\title{
Acquired cystic fibrosis transmembrane conductance regulator protein (CFTR) dysfunction and cigarette smoking
}

\author{
Jonathan Kopel BS
}

\begin{abstract}
Cystic fibrosis (CF) remains a prevalent genetically inherited disease in Caucasian populations. Investigation of the respiratory symptoms which occur in patients with CF helps us understand the pathophysiology of chronic lung disease. Environmental insults, such as cigarette smoke, can reduce the cystic fibrosis transmembrane receptor (CFTR) function or expression leading to an acquired CF phenotype and could contribute to the development and progression of smoking-related lung disease. However, it is uncertain if the acquired CF phenotype can be diagnosed with the same methods, such as the sweat chloride test and the measurement of nasal potential difference, used for genetically-acquired CF. More studies are needed to investigate the prevalence of acquired CFTR dysfunction and the differences between acquired and genetically-inherited CFTR dysfunction. Overall, acquired CFTR dysfunction challenges the distinction between genetic and acquired disorders, suggesting that environmental agents may modulate the functions of genes and the increase risk for pulmonary disease.
\end{abstract}

Keywords: Cystic fibrosis, acquired cystic fibrosis, smoking, mucociliary clearance

\section{CYSTIC FIBROSIS BACKGROUND}

Cystic fibrosis (CF) remains a prevalent genetically inherited disease in Caucasian populations, affecting 1 in 2500 newborns. ${ }^{1}$ It is an autosomal recessive disease caused by mutations in the CF transmembrane conductance regulator gene (CFTR). ${ }^{1}$ Specifically, deletion of phenylalanine at codon 508 , known as $\Delta \mathrm{F} 508$, occurs in $70 \%$ of CF patients; hundreds of rarer CFTR mutations also occur and produce less severe clinical presentations. ${ }^{1}$ The CFTR protein is expressed throughout the body and regulates fluid volume on epithelial surfaces by modulating chloride secretion and sodium absorption. ${ }^{1}$ In CF, CFTR mutations prevent the secretion of chloride ions on epithelial surfaces,

Corresponding author: Jonathan Kopel Contact Information: Jonathan.kopel@ttuhsc.edu DOI: 10.12746/swrccc.v7i30.562 which alters the ionic composition of extracellular fluid and reduces water secretion leading to thickened mucus on airway surfaces. ${ }^{1}$ According to the "low volume" hypothesis, the reduced airways surface volume decreases the mucociliary clearance function to eliminate inhaled bacteria on lung epithelial surfaces. ${ }^{1}$ The increased bacterial load and increased inflammatory response of the lung damages lung tissue and thereby reducing respiratory function. ${ }^{1}$ Furthermore, CF causes meconium ileus, fat soluble vitamin deficiencies, and male infertility. ${ }^{1}$ Despite diagnostic and therapeutic advances, CF patients have a median survival of 50 years. ${ }^{1}$

\section{Cigarette smoking and ACQUiRED CYSTIC FIBROSIS}

Chronic exposure to cigarette smoke damages the upper and lower airways and reduces mucus clearance, which is strongly associated with COPD. ${ }^{2-4}$ COPD includes several clinical manifestations resulting 
from large-airway inflammation and remodeling with increased airway resistance and from alveolar wall destruction with decreased alveolar lung surface area available for gas exchange. ${ }^{5}$ Studies in patients with tobacco smoke related COPD (confirmed homozygote wild-type CFTR genotype) have demonstrated the presence of acquired CFTR dysfunction in some COPD patients and that this persists despite smoking cessation and is associated with chronic bronchitis severity. ${ }^{6}$ Environmental insults, such as cigarette smoke, can reduce CFTR function or expression leading to an acquired CFTR dysfunction, possibly contributing to the development and progression of COPD..$^{2-4}$

The population distribution of sweat chloride levels is unknown and may vary by country and ethnicity. Studies have focused on patients with respiratory symptoms or disease. An epidemiology study at a Brazilian referral CF center examining 5,721 patients (0-85.58 years old) suspected of cystic fibrosis, over a 30 year period, reported that $61.6 \%$ had a sweat chloride level less than $30 \mathrm{mmol} / \mathrm{L}$ chloride, $25.1 \%$ had a sweat chloride level between 30 and $60 \mathrm{mmol} / \mathrm{L}$ chloride, and $13.3 \%$ had a sweat chloride level greater than $60 \mathrm{mmol} / \mathrm{L}$ chloride. ${ }^{7}$ Another study examining 182 patients (0.2-23 years old) at a Northern India hospital with suspected cystic fibrosis with one or more clinical features of cystic fibrosis found $5 \%$ had borderline sweat chloride levels (sweat chloride levels $40-59 \mathrm{mmol} / \mathrm{L}$ chloride) and $22.5 \%$ had elevated sweat chloride levels (>60 mmol/L chloride). ${ }^{8}$ Among current and former smokers, $20-31 \%$ have elevated sweat chloride levels ( $\geq 40 \mathrm{mmol} / \mathrm{L}$ chloride), which indicates a reduced function and expression of CFTR. ${ }^{9,10}$ Similarly, a clinical report examining former or current smokers with COPD (excluding those with CFTR gene mutation) showed $40 \%$ of had an elevated sweat chloride test. ${ }^{10,11}$

Among the various components in cigarette smoke, acrolein and cadmium produce the greatest decrease in CFTR function and expression along the respiratory epithelium. ${ }^{2-4}$ Specifically, acrolein and cadmium produce CFTR dysfunction through reducing CFTR expression and function, destabilizing CFTR proteins, and chemically modifying cysteine and lysine residues..$^{2-4,12-14}$ Similar alterations in
CFTR function and expression were observed with e-cigarette vapors, which contain acrolein and other toxins. ${ }^{15}$ Pathological studies of upper and lower airway epithelial cells demonstrate that cigarette smoke exposure decreases ciliary beating frequency through reduced CFTR and calcium activated chloride channel, which directly inhibits mucociliary clearance. ${ }^{2-4,14}$ Furthermore, studies measuring nasal potential difference and lower airway potential difference and studies with endobronchial biopsies from subjects without CFTR genetic mutations showed significant decreases in CFTR protein function and expression with acute and chronic exposure of cigarette smoke..$^{2-4,16-18}$ Chemical components in cigarette smoke also enter systemic circulation and can produce pancreatitis, infertility, and cachexia. ${ }^{2-4}$ Overall, these studies suggest cigarette smoke oxidants reduce CFTR mRNA transcript expression, accelerate CFTR protein degradation, and alter CFTR channel gating leading to acquired CFTR dysfunction. ${ }^{4}$

However, it is uncertain whether acquired CFTR dysfunction can be diagnosed with the same methods, such as quantitative sweat chloride concentrations and measurement of nasal potential differences, used for genetically inherited cystic fibrosis. ${ }^{19}$ It remains unknown whether acquired CFTR dysfunction in COPD patients increases the severity of cough, sputum production, and deterioration in lung function beyond the usual effects of cigarette smoking alone. Since a significant proportion of COPD patients have been shown to have CFTR dysfunction, this suggests the CFTR protein dysfunction might be an important contributor to the pathogenesis of COPD and other pulmonary diseases. ${ }^{6}$ More studies are needed to investigate clinical or phenotypic expressive differences between a patient population with acquired CFTR dysfunction and one with genetically inherited CF. ${ }^{19}$ For example, smokers with acquired CFTR dysfunction do not typically present with pancreatic enzyme deficiencies or obstruction of the vas deferens seen in those with genetically inherited CF. ${ }^{19}$ Currently, $\mathrm{N}$-acetylcysteine, ivacaftor, and roflumilast can reverse decreases in CFTR function and expression. ${ }^{12,14,20-22}$ However, ivacaftor is effective in CF patients with a less common CFTR gene mutation, G551D, and requires more clinical trials to determine 
whether it will be effective against tobacco smoke induced acquired CFTR dysfunction. ${ }^{19}$ Despite limited therapeutic options, a clinical report examining smoking cessation showed improved CFTR-dependent sweat secretion rate over a 21 -day period. ${ }^{23}$ Overall, acquired CFTR dysfunction challenges the distinction between genetic and acquired disorders and suggests that environmental agents may modulate the function of genes and the risk for disease. ${ }^{19}$

Article citation: Kopel J. Acquired cystic fibrosis transmembrane conductance regulator protein (CFTR) dysfunction and cigarette smoking. The Southwest Respiratory and Critical Care Chronicles 2019;7(30): 43-46

From: The School of Medicine, Texas Tech University Health Sciences Center, Lubbock, Texas

Submitted: $3 / 9 / 2019$

Accepted: 6/19/2019

Reviewer: Adaobi Kanu MD

Conflicts of interest: none

This work is licensed under a Creative Commons Attribution-ShareAlike 4.0 International License.

\section{REFERENCES}

1. Bush A, Davies JC. Cystic fibrosis. Resp Med 2013;355: 315-326.

2. Banks C, Freeman L, Cho DY, et al. Acquired cystic fibrosis transmembrane conductance regulator dysfunction. World J Otorhinolaryngol Head Neck Surg 2018;4(3):193-199.

3. Cho DY, Woodworth BA. Acquired cystic fibrosis transmembrane conductance regulator deficiency. Adv Otorhinolaryngol 2016;79:78-85.

4. Shi J, Li H, Yuan C, et al. Cigarette smoke-induced acquired dysfunction of cystic fibrosis transmembrane conductance regulator in the pathogenesis of chronic obstructive pulmonary disease. Oxid Med Cell Longev 2018;2018:1-13.

5. Tuder RM, Petrache I. Pathogenesis of chronic obstructive pulmonary disease. J Clin Inves 2012;122(8):2749-2755.

6. Raju SV, Solomon GM, Dransfield MT, et al. Acquired cystic fibrosis transmembrane conductance regulator dysfunction in chronic bronchitis and other diseases of mucus clearance. Clin Chest Med 2016;37(1):147-158.

7. Faria AG, Marson FAL, Gomez CCS, et al. Thirty years of sweat chloride testing at one referral center. Frontiers in Ped 2017;5.
8. Raina MA. Assessment of correlation between sweat chloride levels and clinical features of cystic fibrosis patients. J Clin Diag Res 2016.

9. Griesenbach U. Faculty of 1000 evaluation for cigarette smoke induces systemic defects in cystic fibrosis transmembrane conductance regulator function. In. Post-Publication Peer Rev of the Biomed Lit: F1000 ( Faculty of 1000 Ltd); 2015.

10. Raju SV, Jackson PL, Courville CA, et al. Cigarette smoke induces systemic defects in cystic fibrosis transmembrane conductance regulator function. Ameri J Resp Crit Care Med 2013;188(11):1321-1330.

11. Courville CA, Tidwell S, Liu B, et al. Acquired defects in CFTR-dependent $\beta$-adrenergic sweat secretion in chronic obstructive pulmonary disease. Resp Res 2014;15(1):25.

12. Raju SV, Lin VY, Liu L, et al. The cystic fibrosis transmembrane conductance regulator potentiator ivacaftor augments mucociliary clearance abrogating cystic fibrosis transmembrane conductance tegulator inhibition by cigarette smoke. Am J Respir Cell Mol Biol 2017;56(1):99-108.

13. Cantin AM, Hanrahan JW, Bilodeau G, et al. Cystic fibrosis transmembrane conductance regulator function is suppressed in cigarette smokers. Am J Respir Crit Care Med 2006;173(10):1139-1144.

14. Sloane PA, Shastry S, Wilhelm A, et al. A pharmacologic approach to acquired cystic fibrosis transmembrane conductance regulator dysfunction in smoking related lung disease. PloS One 2012;7(6):e39809-e39809.

15. Lin VY, Fain MD, Jackson PL, et al. Vaporized e-cigarette liquids induce ion transport dysfunction in airway epithelia. Am J Respir Cell Mol Biol 2018.

16. Savitski AN, Mesaros C, Blair IA, et al. Secondhand smoke inhibits both $\mathrm{Cl}-$ and $\mathrm{K}+$ conductances in normal human bronchial epithelial cells. Resp Res 2009;10(1):120-120.

17. Kreindler JL, Jackson AD, Kemp PA, et al. Inhibition of chloride secretion in human bronchial epithelial cells by cigarette smoke extract. Amer J Phys 2005;288(5):L894-L902.

18. Clunes LA, Davies CM, Coakley RD, et al. Cigarette smoke exposure induces CFTR internalization and insolubility, leading to airway surface liquid dehydration. FASEB J 2012; 26(2):533-545.

19. CrystalRG.Arethesmoking-induceddiseasesanacquiredform of cystic fibrosis? Am J Respir Crit Care Med 2013;188(11): 1277-1278.

20. Raju SV, Rasmussen L, Sloane PA, et al. Roflumilast reverses CFTR-mediated ion transport dysfunction in cigarette smoke-exposed mice. Resp Res 2017;18(1):173-173.

21. Solomon GM, Fu L, Rowe SM, et al. The therapeutic potential of CFTR modulators for COPD and other airway diseases. Curr Opini in Pharm 2017;34:132-139. 
22. Lambert JA, Raju SV, Tang LP, et al. Cystic fibrosis transmembrane conductance regulator activation by roflumilast contributes to therapeutic benefit in chronic bronchitis. Amer J Resp Cell Molec Biol 2014;50(3):549-558.
23. Courville CA, Raju SV, Liu B, et al. Recovery of acquired cystic fibrosis transmembrane conductance regulator dysfunction after smoking cessation. Amer J Resp Crit Care Med 2015;192(12):1521-1524. 